\title{
Theoretical Analysis of Astronomical Phased Arrays
}

\author{
Stafford Withington, George Saklatavala, and Michael P. \\ Hobson \\ Cavendish Laboratory, JJ Thompson Avenue, Cambridge CB3 OHE, UK \\ E-mail: stafford@mrao.cam.ac.uk
}

\begin{abstract}
Low-noise phased arrays are essential for the next generation of microwave and submillimetre wave astronomy. We analyze their behaviour from a functional perspective, and show that their operation is intimately related to the mathematical theory of frames. No assumptions are made about the orthogonality or linear independence of the synthesised beams. Frame theory allows an unambiguous assessment of whether the outputs of an array can be used to observe a field or brightness distribution within a given class. Image reconstruction is carried out using dual beams. We identify the natural modes of phased arrays, and carry out an analysis of noise. The scheme allows the expectation values, the mean-square fluctuations, and the correlations between fluctuations at the output ports of a phased array to be determined for a source in any state of spatial coherence. Both classical and photoncounting statistics are included. Our model is conceptually powerful, and suggests many simulation and image recovery techniques.
\end{abstract}

Keywords: Phased arrays, planar arrays, imaging arrays, frame theory, astronomical telescopes, therahertz instruments, millimetre-wave instruments, optical modes, coherence 


\section{Introduction}

Recently, there has been a surge of interest in developing phased arrays for radio astronomy. Projects include the Square Kilometer Array (SKA), the Low Frequency Array (LOFAR), the Electronic Multibeam Radio Astronomy Concept (EMBRACE), the Karoo Array Telescope (KAT) [1, 2, 3, 4], and a number of instruments for enhancing the performance of single-dish telescopes. Most of the current projects are aimed constructing phased arrays for microwave astronomy, but as technological capability improves, phased arrays will also be constructed for far infrared and submillimetre wave astronomy [5, 6].

Two types of phased array are of interest: (i) imaging phased arrays, where an array of passive antennas, or coherent receivers, is connected to a beam-forming network such that synthesised beams can be created and swept across the sky; (ii) interferometric phased arrays, where the individual antennas of an aperture synthesis interferometer are equipped with phased arrays such that fringes are formed within the synthesised beams. In this way it is possible to extend the field of view, to observe completely different

regions of the sky simultaneously, to steer the field of view electronically, and to observe spatial frequencies that are not available because the baselines of an interferometer cannot be packed more tightly than the diameters of the individual telescopes.

It is important to appreciate that the synthesised beams of a phased array need not, mathematically speaking, be orthogonal and may even be linearly dependent. Nonorthogonality may be built into a system intentionally as a way of increasing the fidelity with which an image can be reconstructed, or it may arise inadvertently as a consequence of RF coupling and post-processing cross-talk. In some situations, say in the case of interacting planar antennas, it may not even be clear how to distinguish one antenna from another, even before the beam-forming network has been connected.

Despite the considerable importance of phased arrays for astronomy, there is still a lack of information about the principles of operation when the synthesised beams are non-orthogonal, or even linearly dependent, and when noise is included. In this paper we describe the operation of low-noise imaging phased arrays from a functional perspective, and show that information throughput and image recovery are intimately related to the mathematical theory of frames. We also show that it is only necessary to know the synthesised beams in order to calculate the average powers, the correlations between the complex travelling wave amplitudes, the fluctuations in power, and the correlations between the fluctuations in power (the Hanbury Brown-Twiss effect) at the output ports of an array.

The paper provides a powerful conceptual framework for understanding the operation of phased arrays. A key feature of the analysis is that we do not fill the half space between the aperture plane and the sky with a complete set of plane waves, but limit our attention to the natural optical modes of the system. In this way, issues relating to information throughput, sensitivity, noise, correlations, and quantum statistics can be dealt with in a straightforward manner. Indeed, the quantum statistical properties 
of the incoming radiation field can be taken into account, for a source in any state of spatial coherence, and the transition from fully bunched to photon-counting statistics included as the wavelength of operation moves from the microwave range through into the far infrared.

\section{Basic Principles}

In general an imaging phased array comprises a sequence of optical components, an array of single-mode receivers, each of which has a primary beam pattern, and an electrical beam-forming network such that each output port corresponds to a synthesised reception pattern on the sky. The synthesised reception patterns may be static and designed to give optimum sampling on a given class of object, or they may be controlled electrically and swept across the field of view. In the case of radio astronomy, the optical system would be a telescope, the single-mode receivers would be horns or planar antennas coupled to HEMT amplifiers or SIS mixers, and the beam-forming network would be a system of microwave or digital electronics.

The following analysis is based on a generic system comprising an array of $M$ horns and a beam-forming network having $P$ output ports. Each of the $P$ ports is associated with a synthesised reception pattern. For convenience, $\mathcal{A}$ denotes the input reference surface, which is a far-field region of the sky, $\mathcal{B}$ the output ports of the horns, and $\mathcal{C}$ the output ports of the beam-forming network.

When a fully coherent field is incident on the system, a set of travelling waves appears at $\mathcal{B}$, and we shall denote their complex amplitudes by $\left\{y_{m}: m \in 1, \cdots, M\right\}$; also, a set of travelling waves appears at $\mathcal{C}$, and we shall denote their complex amplitudes by $\left\{z_{p}: p \in 1, \cdots, P\right\}$. Because $M$ and $P$ are finite, the complex amplitudes can be assembled into column vectors $\mathbf{y} \in \mathbb{C}^{M}$ and $\mathbf{z} \in \mathbb{C}^{P}$. If $\mathbf{E}(\hat{\mathbf{\Omega}})$ is the plane-wave spectrum of the incident electric field, and $\hat{\Omega}$ a unit radial vector in the aperture plane pointing towards the sky, then it can be shown that because the synthesised reception patterns and incoming field are square-integrable functions, which can be represented by vectors in the Hilbert space of square integral functions over $\mathcal{A}$, the complex amplitude of the travelling wave at port $p$ can be written

$$
z_{p}=\int_{\mathcal{A}} \mathbf{t}_{p}^{*}(\hat{\boldsymbol{\Omega}}) \cdot \mathbf{E}(\hat{\mathbf{\Omega}}) d \boldsymbol{\Omega},
$$

where $\mathbf{t}_{p}(\hat{\boldsymbol{\Omega}})$ is the complex synthesised reception pattern of port $p$. It would be naive to assume, however, that if the array is illuminated by a field having the form $\mathbf{E}(\hat{\mathbf{\Omega}})=\mathbf{t}_{p}(\hat{\mathbf{\Omega}})$, a travelling wave only appears at $p$.

It is instructive to derive a relationship between the synthesised beams and the beams of the primary antennas. If the beam patterns of the primary antennas are denoted by $\mathbf{h}_{m}(\hat{\mathbf{\Omega}})$ then the outputs of the antennas, $y_{m}$, are given by

$$
y_{m}=\int_{\mathcal{A}} \mathbf{h}_{m}^{*}(\hat{\mathbf{\Omega}}) \cdot \mathbf{E}(\hat{\mathbf{\Omega}}) d \mathbf{\Omega},
$$


but the beam-forming network can be described by scattering matrix $\boldsymbol{\Phi}$, having elements $\phi_{p m}$, where

$$
z_{p}=\sum_{m} \phi_{p m} y_{m},
$$

and therefore substituting (2) in (3),

$$
z_{p}=\int_{\mathcal{A}} \sum_{m} \phi_{p m} \mathbf{h}_{m}^{*}(\hat{\boldsymbol{\Omega}}) \cdot \mathbf{E}(\hat{\boldsymbol{\Omega}}) d \boldsymbol{\Omega},
$$

which can be cast into the form of (11) by defining

$$
\mathbf{t}_{p}(\hat{\mathbf{\Omega}})=\sum_{m} \phi_{p m}^{*} \mathbf{h}_{m}(\hat{\mathbf{\Omega}}) .
$$

As expected, the synthesised reception patterns are weighted linear combinations of the primary antenna patterns.

Even when the primary beams are orthogonal, and identical, the synthesised beams do not have to be orthogonal, because

$$
\int_{\mathcal{A}} \mathbf{t}_{p}^{*}(\hat{\boldsymbol{\Omega}}) \cdot \mathbf{t}_{p^{\prime}}(\hat{\boldsymbol{\Omega}}) d \boldsymbol{\Omega}=k \sum_{m} \phi_{p m} \phi_{p^{\prime} m}^{*},
$$

where (5) has been used, $k$ is a constant that derives from the inner products of the primary antenna patterns,

$$
\int_{\mathcal{A}} \mathbf{h}_{m}^{*}(\hat{\boldsymbol{\Omega}}) \cdot \mathbf{h}_{m^{\prime}}(\hat{\boldsymbol{\Omega}}) d \boldsymbol{\Omega}=k \delta_{m m^{\prime}},
$$

and there is no restriction on the orthogonality of the $\phi_{p m}$. (6) can be written in the form of a matrix equation:

$$
\int_{\mathcal{A}} \mathbf{t}_{p}^{*}(\hat{\boldsymbol{\Omega}}) \cdot \mathbf{t}_{p^{\prime}}(\hat{\boldsymbol{\Omega}}) d \boldsymbol{\Omega}=k\left[\boldsymbol{\Phi} \Phi^{\dagger}\right]_{p p^{\prime}} .
$$

If $M$ of the columns of $\boldsymbol{\Phi}^{\dagger}: \mathbb{C}^{P} \rightarrow \mathbb{C}^{M}$ are linearly independent, and $P>M$, then the complete set of columns constitutes an over-complete basis, in the sense that there are $P$ vectors in an $M$-dimensional space. In this case, except trivially when certain ports are not connected, the synthesised reception patterns are not orthogonal, because $\boldsymbol{\Phi} \Phi^{\dagger} \neq \mathbf{D}$, where $\mathbf{D}$ is a diagonal matrix of dimension $P$; indeed, the synthesised reception patterns are linearly dependent. Contrariwise, the columns of $\boldsymbol{\Phi}^{\dagger}$ comprise an under-complete basis if $P<M$, and again we may have $\boldsymbol{\Phi} \boldsymbol{\Phi}^{\dagger} \neq \mathbf{D}$. In the case where $\boldsymbol{\Phi}$ is unitary, $\boldsymbol{\Phi} \boldsymbol{\Phi}^{\dagger}=\mathbf{I}_{P}$, where $\mathbf{I}_{P}$ is the identity matrix of dimension $P$, and the primary beam patterns are orthogonal, the synthesised reception patterns are orthogonal. We conclude that, except in the simplest of scenarios, the synthesised reception patterns are unlikely to be orthogonal,

$$
\int_{\mathcal{A}} \mathbf{t}_{p}^{*}(\hat{\boldsymbol{\Omega}}) \cdot \mathbf{t}_{p^{\prime}}(\hat{\boldsymbol{\Omega}}) d \boldsymbol{\Omega} \neq k D_{p, p^{\prime}}
$$

and may even be linearly dependent. According to (1) and (9), travelling waves will generally appear at more than one port even when the incident field has the form of one of the synthesised reception patterns: $\mathbf{E}(\hat{\boldsymbol{\Omega}})=\mathbf{t}_{p^{\prime}}(\hat{\boldsymbol{\Omega}})$. 
(5) indicates that the synthesised beams are weighted linear combinations of the primary antenna patterns; or conversely, that there must be sufficient antennas of the correct form, such that all of the required synthesised reception patterns, are spanned, mathematically speaking, by the primary antenna patterns. In the case of real systems, the primary beams $\mathbf{h}_{m}(\hat{\boldsymbol{\Omega}}): m \in\{1, \cdots, M\}$, which are the individual beams in the presence of all of the antennas, including scattering, are likely to be different to one another; for example, the antennas at the edge of an array will have different beam patterns to those in the middle. In addition, the primary beams may have far-out sidelobes, which can couple to warm radiating objects such as the ground. Ideally, it must be possible to span all possible synthesised beams, as the source moves across the sky, including the requirement that the synthesised beams must be near zero outside of some field of view. If nulling is not achieved, high noise temperatures, which may be a strong function of $\boldsymbol{\Phi}$, may result. Low noise temperatures can only be achieved by ensuring that the reception patterns of the primary antennas can only couple to low-noise regions of the sky, or, formally speaking, that there are many more degrees of freedom in the system than are needed to simply create the main lobes of the synthesised beams.

In astronomical applications, phased arrays are used to image incoherent or, in the case of celestial masers, partially coherent fields. It is convenient, therefore, to introduce correlation dyadics. Define the correlation dyadic of the incident field according to

$$
\overline{\overline{\mathbf{E}}}\left(\hat{\Omega}_{1}, \hat{\Omega}_{2}\right)=\left\langle\mathbf{E}\left(\hat{\Omega}_{1}\right) \hat{\mathbf{E}}^{*}\left(\hat{\Omega}_{2}\right)\right\rangle,
$$

where $\mathbf{E}(\hat{\mathbf{\Omega}})$ is the complex analytic representation of the quasi-monochromatic electric field, and \langle\rangle denotes the ensemble average. The final result can be integrated with respect to frequency to calculate broadband behaviour, but we do not show frequency dependence explicitly. We shall assume throughout that the electric-field is normalized to the square root of the impedance of free space so that the elements of $\overline{\overline{\mathbf{E}}}\left(\hat{\boldsymbol{\Omega}}_{1}, \hat{\Omega}_{2}\right)$ have the units of $\mathrm{Wm}^{-2} \mathrm{Sr}^{-2} \mathrm{~Hz}^{-1}$. The rank 2 tensor $\overline{\overline{\mathbf{E}}}\left(\hat{\boldsymbol{\Omega}}_{1}, \hat{\boldsymbol{\Omega}}_{2}\right)$ contains complete information about the correlations between all pairs of transverse vector field components for any two points on the sky. Once the correlation dyadic is known, all classical measures of coherence follow.

The correlation between the travelling wave amplitudes at any two output ports is given by $\left\langle z_{p} z_{p^{\prime}}^{*}\right\rangle$, or in matrix form $\mathbf{Z}=\left\langle\mathbf{z z}^{\dagger}\right\rangle \in \mathbb{C}^{P \times P}$. The elements of $\mathbf{Z}$ can be found by using (10) and (10):

$$
Z_{p p^{\prime}}=\int_{\mathcal{A}} \int_{\mathcal{A}} \mathbf{t}_{p}^{*}\left(\hat{\boldsymbol{\Omega}}_{1}\right) \cdot \overline{\overline{\mathbf{E}}}\left(\hat{\boldsymbol{\Omega}}_{1}, \hat{\boldsymbol{\Omega}}_{2}\right) \cdot \mathbf{t}_{p^{\prime}}\left(\boldsymbol{\Omega}_{2}\right) d \boldsymbol{\Omega}_{1} d \boldsymbol{\Omega}_{2},
$$

and because the the travelling wave amplitudes are normalized to the square root of impedance, the elements of $\mathbf{Z}$ have the units $\mathrm{WHz}^{-1}$. According to (11) the correlations at the outputs are merely the matrix elements of the source coherence tensor with respect to the synthesised beams.

In the case of spatially incoherent, but not necessarily unpolarized sources,

$$
\overline{\overline{\mathbf{E}}}\left(\hat{\Omega}_{1}, \hat{\Omega}_{2}\right)=\overline{\overline{\mathbf{B}}}\left(\hat{\Omega}_{1}\right) \delta\left(\hat{\Omega}_{1}-\hat{\Omega}_{2}\right),
$$


where $\overline{\overline{\mathbf{B}}}\left(\hat{\mathbf{\Omega}}_{1}\right)$ contains information about the polarisation of the source, which can be projected onto Pauli-spin matrices or spin-weighted spherical harmonics. $\overline{\overline{\mathbf{B}}}\left(\hat{\boldsymbol{\Omega}}_{1}\right)$ is a brightness tensor because it has units $\mathrm{Wm}^{-2} \mathrm{Sr}^{-1} \mathrm{~Hz}^{-1}$. In the case of incoherent and unpolarised sources, (12) becomes

$$
\overline{\overline{\mathbf{E}}}\left(\hat{\mathbf{\Omega}}_{1}, \hat{\mathbf{\Omega}}_{2}\right)=B\left(\hat{\mathbf{\Omega}}_{1}\right) \overline{\overline{\mathbf{I}}} \delta\left(\hat{\mathbf{\Omega}}_{1}-\hat{\mathbf{\Omega}}_{2}\right)
$$

where $\overline{\overline{\mathbf{I}}}$ is the unit dyad, and $B\left(\hat{\boldsymbol{\Omega}}_{1}\right)$ the brightness of each polarisation in the direction of $\hat{\Omega}_{1}$.

(13) can be expressed in terms of a brightness temperature, $T_{b}(\hat{\boldsymbol{\Omega}})$, through

$$
\overline{\overline{\mathbf{E}}}\left(\hat{\boldsymbol{\Omega}}_{1}, \hat{\boldsymbol{\Omega}}_{2}\right)=\frac{h \nu^{3}}{c^{2}} \frac{1}{\exp \left[h \nu / k T_{b}\left(\hat{\boldsymbol{\Omega}}_{1}\right)\right]-1} \overline{\overline{\mathbf{I}}} \delta\left(\hat{\boldsymbol{\Omega}}_{1}-\hat{\boldsymbol{\Omega}}_{2}\right) .
$$

(14) predicts an infinite correlation dyadic when $\hat{\Omega}_{1}=\hat{\Omega}_{2}$, which occurs because an infinitely small coherence area requires an infinitely large number of radiators to be packed into every finite region of the sky. Nevertheless, (14) behaves correctly when integrated with respect to antenna power patterns, because antenna power patterns, even in the multimode case, have finite coherence areas.

Numerous sources can be modelled in this way, for example, a polarized source can be combined with unpolarized emission from the atmosphere having brightness temperature $T_{s}$, to give

$$
\overline{\overline{\mathbf{E}}}\left(\hat{\boldsymbol{\Omega}}_{1}, \hat{\boldsymbol{\Omega}}_{2}\right)=\left[\frac{h \nu^{3}}{c^{2}} \frac{1}{\exp \left[h \nu / k T_{s}\right]-1} \overline{\overline{\mathbf{I}}}+\overline{\overline{\mathbf{B}}}\left(\hat{\boldsymbol{\Omega}}_{1}\right)\right] \delta\left(\hat{\boldsymbol{\Omega}}_{1}-\hat{\boldsymbol{\Omega}}_{2}\right) .
$$

Some care is needed when $T_{s}$ is not uniform because the source is in the far field of the array whereas the atmosphere is usually in in the near field; these effects can be taken into account, but we shall not do so here.

Now illuminate a phased array with an unpolarised, incoherent source. Substituting (13) in (11), the elements of the coherence matrix become

$$
Z_{p p^{\prime}}=\int_{\mathcal{A}} B(\hat{\boldsymbol{\Omega}}) \mathbf{t}_{p}^{*}(\hat{\mathbf{\Omega}}) \cdot \mathbf{t}_{p^{\prime}}(\hat{\boldsymbol{\Omega}}) d \boldsymbol{\Omega}
$$

the diagonal elements of which correspond to coupling the incoming radiation field to the synthesised power patterns, $K_{p}(\hat{\boldsymbol{\Omega}})=\mathbf{t}_{p}^{*}(\hat{\boldsymbol{\Omega}}) \cdot \mathbf{t}_{p}(\hat{\boldsymbol{\Omega}})$, giving

$$
Z_{p p}=\int_{\mathcal{A}} B(\hat{\boldsymbol{\Omega}}) K_{p}(\hat{\boldsymbol{\Omega}}) d \boldsymbol{\Omega} .
$$

The functions $K_{p}(\hat{\boldsymbol{\Omega}})$ are direction-dependent effective areas, and we could use $K_{p}(\hat{\boldsymbol{\Omega}})=$ $A_{p} P_{p}(\hat{\Omega})$, where $A_{p}$ is the effective area of the $p^{\prime}$ th synthesised beam in the most receptive direction, and $P_{p}(\hat{\boldsymbol{\Omega}})$ is the dimensionless power pattern. Substituting the Planck formula in (17), and assuming a uniform sky temperature, gives

$$
\begin{aligned}
Z_{p p} & =\int_{\mathcal{A}} \frac{h \nu^{3}}{c^{2}} \frac{1}{\exp \left[h \nu / k T_{s}\right]-1} A_{p} P_{p}(\hat{\boldsymbol{\Omega}}) d \boldsymbol{\Omega} \\
& =\frac{A_{p} \Omega_{p}}{\lambda^{2}} \frac{h \nu}{\exp \left[h \nu / k T_{s}\right]-1} \\
& =\kappa_{p} \frac{h \nu}{\exp \left[h \nu / k T_{s}\right]-1},
\end{aligned}
$$


where $\kappa_{p}=A_{p} \Omega_{p} / \lambda^{2}$ is a coupling factor. For orthogonal primary beams, and a unitary beam-forming network, $A_{p} \Omega_{p} / \lambda^{2}$ can be regarded as the number of modes in the beam, which is unity, and we arrive at the expected spectral power in the travelling wave. Generally, however, for a phased array, $A_{p} \Omega_{p} / \lambda^{2}<1$. Certainly $\sum_{p} A_{p} \Omega_{p} / \lambda^{2} \leq M$, because the total power cannot exceed the modal throughput of the primary antennas.

(16) shows that because the synthesised reception patterns are generally not orthogonal the travelling waves at the output ports are correlated, even when the sky brightness is uniform. If the source is not uniform, correlations exist even when the synthesised beams are orthogonal, which corresponds to the usual case of interferometry. Thus, each port of a phased array records the incident flux in the usual manner, but because the beams are not necessarily orthogonal, and may even be linearly dependent, the travelling waves at the output ports may be correlated, and the correlations will be more complicated than in the usual case of interferometry.

\section{Information Throughput and Image Recovery}

Consider information throughput and image recovery. Represent the primary physical quantities as abstract vectors. Because the incoming field, $\mathbf{E}(\hat{\mathbf{\Omega}})$, is square integrable over the appropriate region of the sky, $\mathcal{A}$, it can be represented by a vector $|\mathbf{E}\rangle$ in Hilbert space $\mathbb{H}$. Regions having different shapes and sizes correspond to different Hilbert spaces. In the case of realizable phased arrays, where the numbers of ports and primary antennas are finite, the measurable quantities $\mathbf{y}$ and $\mathbf{z}$ are finite-dimensional vectors, $\mathbf{y} \in \mathbb{C}^{M}$ and $\mathbf{z} \in \mathbb{C}^{P}$, but because we wish to draw attention to the relationship with frame theory, and to emphasize the role of convergence, we use the more general representations $|\mathbf{y}\rangle \in \ell^{2}$ and $|\mathbf{z}\rangle \in \ell^{2}$, where $\ell^{2}$ is the space of square-summable complex sequences. These definitions lead to two operators, one of which, $\hat{\mathbf{H}}: \mathbb{H} \rightarrow \ell^{2}$, maps the incoming electric field onto the outputs of the horns, and the other $\hat{\boldsymbol{\Phi}}: \ell^{2} \rightarrow \ell^{2}$ maps the outputs of the horns onto the outputs of the beam-forming network. These individual operators can be combined into a single composite operator $\hat{\mathbf{T}}=\hat{\mathbf{\Phi}} \hat{\mathbf{H}}: \mathbb{H} \rightarrow \ell^{2}$, which describes the system as a whole.

The operation of phased arrays is intimately related to the mathematical theory

of frames. Suppose that we have some general monochromatic field $|\mathbf{E}\rangle$, and that we determine the inner products with respect to a set of basis vectors $\mathbb{T}=\left\{\left|\mathbf{t}_{p}\right\rangle, p \in\right.$ $1, \cdots, P\}: z_{p}=\left\langle\mathbf{t}_{p} \mid \mathbf{E}\right\rangle . P$ can extend to infinity, and we do not make any assumptions about the orthonormality or linear independence of $\mathbb{T}$. Under what circumstances can the original vector $|\mathbf{E}\rangle$, which represents a continuous function, be recovered unambiguously from a discrete, possibly countable, set of complex coefficients, and how can this be achieved? In the context of phased arrays, we are asking under what circumstances can the form of an incident electric field be recovered unambiguously from the complex travelling-wave outputs.

Frame theory [7, 8, 9] proceeds as follows. Evaluate the square moduli of the inner products between $\mathbb{T}$ and any general vector, $|\mathbf{E}\rangle \in \mathbb{H}$, and sum the results. If there are 
two non-zero constants $A$ and $B$ such that $0<A<\infty$ and $0<B<\infty$, and

$$
A\|\mathbf{E}\|^{2} \leq \| \hat{\mathbf{T}}|\mathbf{E}\rangle\left\|^{2} \leq B\right\| \mathbf{E} \|^{2},
$$

which can also be written

$$
A\|\mathbf{E}\|^{2} \leq \sum_{p}\left|\left\langle\mathbf{t}_{p} \mid \mathbf{E}\right\rangle_{\mathbb{H}}\right|^{2} \leq B\|\mathbf{E}\|^{2},
$$

$\forall|\mathbf{E}\rangle \in \mathbb{H}$, then the basis set $\mathbb{T}$ is called a frame with respect to $\mathbb{H}$.

Notice the strict use of inequalities in the allowable values of $A$ and $B$. In the case where $A \approx B$, the frame is called a tight frame because the inner products for all $|\mathbf{E}\rangle \in \mathbb{H}$ lie within some small range, and the dynamic range needed for inversion is small. When the original basis is orthonormal, the frame bounds, $A$ and $B$, are equal, as can be appreciated by inserting $|\mathbf{E}\rangle=\left|\mathbf{t}_{p^{\prime}}\right\rangle$ in (20). If the frame is normalized, $A$ is a measure of the redundancy in the frame. If a basis set constitutes a frame, then it can be shown through (19) alone that the electric field can be recovered unambiguously from the inner products, and the more tightly bound the frame, the more tightly bound the inverse, and the more stable the image recovery process. The frame condition, (19), is completely general, and applies for any continuous function, even though an infinite number of possibly linearly dependent basis functions may be used. Phased arrays have a finite number of ports, but can nevertheless span spatially band-limited functions having finite support, and can therefore form frames with respect to fields carrying finite information.

Because the synthesised beam patterns of a phased array may be non-orthogonal, and even linearly dependent, the recovery of the original field, through an operator we shall call $\hat{\mathbf{T}}^{-1}$, is best implemented by the introduction of dual vectors, which correspond to dual beams. The dual vectors $\left.\widetilde{\mathbf{t}}_{p}\right\rangle$ of any given frame $\mathbb{T}$, with respect to Hilbert space $\mathbb{H}$, are given by

$$
\left|\widetilde{\mathbf{t}}_{p}\right\rangle=\hat{\mathbf{S}}^{-1}\left|\mathbf{t}_{p}\right\rangle,
$$

where $\hat{\mathbf{S}}=\hat{\mathbf{T}}^{\dagger} \hat{\mathbf{T}}$ is non-singular, and can therefore be inverted. The dual basis set, which we shall call $\widetilde{\mathbb{T}}=\left\{\left|\widetilde{\mathbf{t}}_{p}\right\rangle, p \in 1, \cdots, P\right\}$, has the same degree of completeness as the original frame, $\mathbb{T}$, and therefore it too constitutes a frame with respect to $\mathbb{H}$. Indeed, two representations of any general $|\mathbf{E}\rangle$ are possible:

$$
\left.|\mathbf{E}\rangle=\sum_{p}\left\langle\mathbf{t}_{p} \mid \mathbf{E}\right\rangle \widetilde{\mathbf{t}}_{p}\right\rangle \quad|\mathbf{E}\rangle=\sum_{p}\left\langle\widetilde{\mathbf{t}}_{p} \mid \mathbf{E}\right\rangle\left|\mathbf{t}_{p}\right\rangle
$$

(22) shows that if one calculates a set of coefficients by taking the inner products with a frame, then one inverts the process by reconstructing the field using the dual vectors. In the case where the basis vectors are perfectly complete with respect to $\mathbb{H}$, but not necessarily orthogonal, the basis is called a Riesz basis, and the basis set $\mathbb{T}$ and dual set $\widetilde{\mathbb{T}}$ are biorthogonal: $\left\langle\widetilde{\mathbf{t}}_{p} \mid \mathbf{t}_{p^{\prime}}\right\rangle=\delta_{p p^{\prime}}: \forall p, p^{\prime} \in 1, \cdots, P$.

In the case where the basis vectors do not constitute a frame, but an attempt is made to reconstruct the original field vector using the duals,

$$
\left|\mathbf{E}^{\prime}\right\rangle=\sum_{p}\left\langle\mathbf{t}_{p} \mid \mathbf{E}\right\rangle\left|\widetilde{\mathbf{t}}_{p}\right\rangle,
$$


the reconstructed vector $\left|\mathbf{E}^{\prime}\right\rangle$ cannot, for all vectors in $\mathbb{H}$, be the same as the original vector $|\mathbf{E}\rangle$. It can be shown, however, that the error vector $|\mathbf{E}\rangle-\left|\mathbf{E}^{\prime}\right\rangle$ is orthogonal to the basis vectors. Consequently, $\left|\mathbf{E}^{\prime}\right\rangle$ is the orthogonal projection of $|\mathbf{E}\rangle$ onto $\mathbb{S}$, the subspace spanned by the under complete set of basis vectors. In other words the solution is as close as possible to the original field vector to within the degrees of freedom available.

The relevance to phased arrays is clear; one can measure the complex outputs of a phased array, and if the synthesised reception patterns constitute a frame with respect to the Hilbert space defined by the shape, extent, and illumination of the input reference surface, then the continuous, coherent, incoming field can be reconstructed completely from the complex travelling wave amplitudes at the output ports. If the reception patterns do not constitute a frame, reconstruction leads to the least square fit that is consistent with the degrees of freedom to which the phased array is sensitive. If the field of interest has passed through an optical system, which can only ever transmit a finite number of modes, then frames can in principle be formed; if the input surface corresponds to the sky, frames are not possible, because the dimensionality of a field, even over a finite region, is infinite. It is possible, however, to form a frame with respect to some given class of object, as will be discussed. It is also possible to synthesize more and more beams to tighten a frame, thereby increasing the stability of the image recovery process. Central to this model is the notion of dual beams. Every synthesised beam has a dual beam, and the concept of dual beams is central to understanding the optical physics of phased arrays, and the image recovery process.

To this point it has been assumed that the incoming field is fully coherent, but in the case of astronomical phased arrays the incoming field is usually incoherent, and sometimes only the powers are measured at $\mathcal{C}$. According to (16), the self and cross correlations at the output ports of a phased array, for an incoherent, unpolarized source are given by

$$
Z_{p p^{\prime}}=\int_{\mathcal{A}} B(\hat{\mathbf{\Omega}}) S_{p p^{\prime}}^{*}(\hat{\mathbf{\Omega}}) d \mathbf{\Omega}
$$

where

$$
S_{p p^{\prime}}^{*}(\hat{\mathbf{\Omega}})=\mathbf{t}_{p}^{*}(\hat{\mathbf{\Omega}}) \cdot \mathbf{t}_{p^{\prime}}(\hat{\mathbf{\Omega}}),
$$

but according to (24) the elements of the correlation matrix are merely the inner products of $B(\hat{\boldsymbol{\Omega}})$ with respect to $S_{p p^{\prime}}(\hat{\boldsymbol{\Omega}}) \forall p, p^{\prime} \in\{1, \cdots, P\}$. Hence once again, frame theory can be used to determine the degree to which $B(\hat{\boldsymbol{\Omega}})$ can be recovered from the correlations between the travelling waves at $\mathcal{C}$. The case were only the powers are measured, $Z_{p p} \forall p \in\{1, \cdots, P\}$, is a special case of (24), and corresponds to determining the degree to which the synthesised power patterns span the source brightness distributions of interest. It seems, therefore, that frames can be defined for either fully coherent fields with correlation measurement, for incoherent fields with correlation measurement, or for incoherent fields with only power measurement. We shall call these possibilities field frames, interferometric frames, and intensity frames 
respectively. Note that the term interferometric does not necessarily imply that an aperture synthesis interferometer is being used, but merely that the correlations between the travelling waves at the outputs are measured. Each of these frames can be associated with dual beams of some kind. In fact, it is straightforward to formulate a more general theory, and ask whether a set of synthesised beams forms a frame with respect to the full coherence tensor field of the incoming radiation. This more general theory has interesting applications, but we shall not described it here.

Suppose that the goal is to reconstruct the intensity distribution of an astronomical source, and one needs to know whether the basis $S_{p p^{\prime}}(\hat{\boldsymbol{\Omega}}) \forall p, p^{\prime} \in\{1, \cdots, P\}$ forms an interferometric frame. There is a problem, however, because in assuming that the source is spatially incoherent, we have tacitly assumed that the brightness distribution is a member of an infinite dimensional space. To answer the question correctly, we need to ask whether the phased array is suitable for recovering brightness from the vector space of the brightness distributions of interest. One approach is to describe the range of possible brightness distributions as a finite, weighted linear combination of basis functions, $\psi_{n}(\hat{\mathbf{\Omega}}): n \in\{1, \cdots, N\}$. These functions could, for example, be radial basis functions, or delta functions at certain sample points. The functions need not correspond to a single region, but could correspond to different regions of the sky that need to be imaged simultaneously.

The intensity on the sky, according to our chosen class, can be written

$$
B(\hat{\mathbf{\Omega}})=\sum_{n} a_{n} \psi_{n}(\hat{\mathbf{\Omega}})
$$

and therefore, according to (24), the correlations between the travelling waves at the output ports become

$$
Z_{p p^{\prime}}=\sum_{n} a_{n} F_{p p^{\prime}, n}
$$

where

$$
F_{p p^{\prime}, n}=\int_{\mathcal{A}} S_{p p^{\prime}}^{*}(\hat{\mathbf{\Omega}}) \psi_{n}(\hat{\mathbf{\Omega}}) d \boldsymbol{\Omega} .
$$

Defining the matrix $\mathbf{F}$ whose elements are $F_{q, n}$, where $p p^{\prime}$ is now indexed by the single integer $q$, the frame condition (19) becomes

$$
A \leq \mathbf{a}^{\dagger} \mathbf{F}^{\dagger} \mathbf{F a} \leq B \quad \forall \mathbf{a} \in \mathbb{C}^{N} .
$$

(29) has the Hermitian form, and it is well known that the stationary values of the Hermitian form occur when the unit vector a points in the same directions as the eigenvectors of $\mathbf{F}^{\dagger} \mathbf{F}$, with the stationary values being the corresponding eigenvalues. $A$ and $B$, and hence the tightness of the frame, can be determined by finding the largest and smallest eigenvalues of the Hermitian operator $\mathbf{F}^{\dagger} \mathbf{F}$, which can be found analytically or numerically. Although, the operator $\mathbf{F}^{\dagger} \mathbf{F}$ maps a finite dimensional space onto itself, the mapping passes through a space having infinite dimensions and therefore the integrals in (28) should be evaluated analytically if at all possible. The operator $\mathbf{F}^{\dagger} \mathbf{F}$ simply maps the intensity distribution coefficients of the source onto the measured quantities 
at the output ports of the array and then back again onto the coefficients. If the basis functions $S_{p p^{\prime}}(\hat{\boldsymbol{\Omega}})$ do not span all possible intensity distributions, information is lost when an observation is made, and it is not possible to recover complete information about the source.

In the case where the basis functions correspond to sample points $\hat{\boldsymbol{\Omega}}_{n}$, we have $\psi_{n}(\hat{\boldsymbol{\Omega}})=\delta\left(\hat{\boldsymbol{\Omega}}-\boldsymbol{\Omega}_{n}\right)$ and $F_{p p^{\prime}, n}=S_{p p^{\prime}}^{*}\left(\hat{\boldsymbol{\Omega}}_{n}\right)$. Although, in practice, a frame cannot be formed that reproduces the sky brightness at every one of an infinite number of points, a frame can be formed with respect to a finite number of sample points. Also, shapelets [10, 11, 12], which are essentially Gaussian Hermite and Gaussian Laguerre polynomials, are now being used to parameterize the brightness distributions of astronomical sources, such as galaxies. It would therefore be possible to check whether an imaging phased array forms a frame with respect to a set of shapelets, and to recover the shapelet coefficients directly from observations.

\section{Natural Modes of Phased Arrays}

Superficially speaking, the natural modes of a phased array are those orthogonal field distributions that can pass through the horns and beam-forming network with high efficiency. When the synthesised beams are linearly dependent, or even if they are simply non-orthogonal, the forms of the natural modes are not directly known. The natural modes are, however, central to understanding noise and correlations, to understanding quantum statistical behaviour, which is important at sub-millimetre wavelengths, and to understanding the operation of interferometric phased arrays.

Suppose that a telescope is equipped with an imaging phased array. As described previously, the process of projecting a coherent field onto the complex travelling wave amplitudes at the output ports is described by the mapping $\mathbf{T}:|\mathbf{x}\rangle \longmapsto|\mathbf{z}\rangle$ as $\mathbf{T}: \mathbb{H} \rightarrow \ell^{2}$. The phased array acts as a linear operator between two Hilbert spaces: the space of square integrable functions over the input reference surface, and the space of square summable complex sequences. Because the operator maps between two different Hilbert spaces, it does not make sense to search for the eigenfunctions of such an operator in an attempt to find the natural modes.

For any real system, it is certainly known that every incoming field carries a finite amount of power, and the associated complex travelling waves at the output ports carry a finite amount of power: $\mathbf{T}$ is therefore bounded. More importantly, a real system can only transmit a limited amount of information, because a finite number of primary antennas is used: $\mathbf{T}$ is therefore Hilbert-Schmidt. It follows that the integral operator that maps the incoming field distribution onto the output ports can be written in the form $[13$ ]

$$
z_{p}=\int_{\mathcal{A}} \sum_{i} \sigma_{i} \mathbf{U}_{i}^{p} \mathbf{V}_{i}^{*}(\hat{\boldsymbol{\Omega}}) \cdot \mathbf{E}(\hat{\boldsymbol{\Omega}}) d \boldsymbol{\Omega} .
$$

It is a feature of the Hilbert-Schmidt decomposition that $\left\{\mathbf{V}_{i}(\hat{\mathbf{\Omega}}): i \in 1, \cdots, P\right\}$ and $\left\{\mathbf{U}_{i}^{p}: p \in 1, \cdots, P ; i \in 1, \cdots, P\right\}$ are orthogonal sets and therefore, according to 
(30), the operation of a phased array can be regarded as first mapping the incoming field onto the beams, $\mathbf{V}_{i}(\hat{\mathbf{\Omega}})$, scaling by the singular values, $\sigma_{i}$, and reconstructing the complex travelling wave amplitudes at the output ports through the basis vectors $\mathbf{U}_{i}^{p}$. Those reception patterns, $\mathbf{V}_{i}(\hat{\mathbf{\Omega}})$, associated with non-zero singular values span the field distributions at the input to which the phased array is sensitive, and those output vectors $\mathbf{U}_{i}^{p}$, associated with non-zero singular values span the vectors at the output to which the phased array can couple. These are the natural modes of a phased array, and we shall call them the eigenfields, by analogy with bolometric interferometers [14]. In the case of fields that have been discretised for numerical calculation, $\mathbf{V}_{i}(\hat{\mathbf{\Omega}}), \mathbf{U}_{i}^{p}$, and $\sigma_{i}$ correspond to the singular vectors and singular values of the singular value decomposition of the phased array's transmission matrix $\mathbf{T}$.

It can be shown, [14], although we shall not do so here, that the eigenfields on the sky are the eigenfunctions of the Hermitian operator that maps the sky field onto the travelling waves at the output ports, and then back again onto the sky in a time reversed manner. The input eigenfields are those field distributions that remain unchanged in form after this complete round trip. Likewise, the output eigenfields are the eigenvectors of the Hermitian operator that maps the travelling waves at the output ports onto the sky in a time reversed manner, and then forward again to the output ports. The output eigenfields are those discrete vectors that can make this round trip unchanged in form.

The eigenfields have many unique properties. First, they represent the primary paths by which information can pass from the sky to the output ports. The input eigenfields on the sky are mutually orthogonal, even though the synthesised beams may be non-orthogonal or linearly dependent. The output eigenfields, which are actually discrete vectors in this case, are also mutually orthogonal. Secondly, the number of singular values, $\sigma_{i}$, greater than some threshold is the modal throughput of the system. Thirdly, if two phased arrays are placed side-by-side, with the intention of creating an aperture synthesis interferometer, the input eigenfields having non-zero singular values associated with the different telescopes are mutually orthogonal[14], which makes them ideal for analysing the behaviour of interferometers.

Now consider how the concepts of frames and eigenfields can be used to construct powerful models of imaging phased arrays. The correlations at the output ports can be

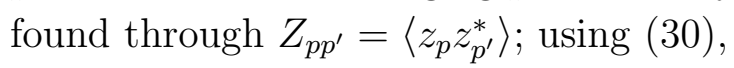

$$
Z_{p p^{\prime}}=\sum_{i} \sum_{i^{\prime}} \sigma_{i} \sigma_{i^{\prime}} \mathbf{U}_{i}^{p} \mathbf{U}_{i^{\prime}}^{p^{\prime}} \int_{\mathcal{A}} \int_{\mathcal{A}} \mathbf{V}_{i}^{*}\left(\hat{\boldsymbol{\Omega}}_{1}\right) \cdot \overline{\bar{E}}\left(\hat{\boldsymbol{\Omega}}_{1}, \hat{\boldsymbol{\Omega}}_{2}\right) \cdot \mathbf{V}_{i^{\prime}}\left(\hat{\boldsymbol{\Omega}}_{2}\right) d \boldsymbol{\Omega}_{1} d \boldsymbol{\Omega}_{2},(31)
$$

which is equivalent to (11), but with the array described in terms of its natural modes. (31) describes the projection of the coherence tensor of the source onto the input eigenfields, the eigenfield coefficients are then multiplied by the singular values, and the correlations at the output ports assembled through the output eigenfields. It is interesting to observe that if a phased array is illuminated by an incoherent, unpolarized, 
spatially uniform source, then using (14) with a uniform temperature $T_{s}$, and (31), gives

$$
Z_{p p^{\prime}}=\frac{h \nu}{\exp [h \nu / k T]-1} \sum_{i} \sigma_{i}^{2} \mathbf{U}_{i}^{p} \mathbf{U}_{i}^{p^{\prime} *},
$$

where we have followed a procedure similar to that described by (18). (32) shows that the correlations at the output are merely those associated with the incoherent excitation of the output eigenfields. Indeed the total power, $W$, becomes

$$
W=\sum_{p} Z_{p p}=\frac{h \nu}{\exp [h \nu / k T]-1} \sum_{i} \sigma_{i}^{2},
$$

where we have used the orthogonality of the output eigenfields. Comparing with (18),

$$
\sum_{p} A_{p} \Omega_{p} / \lambda^{2}=\sum_{p} \sigma_{p}^{2} \leq M .
$$

The total throughput is determined by the number of singular values significantly greater than some threshold, usually determined by the noise. (34) can be regarded as a statement about the modal throughput of a complete array; an important performance metric of phased arrays can therefore be determined from the synthesised beams alone.

\section{Noise}

Consider the case where the primary antennas and beam forming network are are made up of passive components, such as a planar array and microstrip coupling network. What noise sources appear at the output as a consequence of the losses? Because the signal is uncorrelated with the internally generated noise, the correlation matrix at the output can be written $\mathbf{Z}^{\prime}=\mathbf{Z}+\mathbf{Z}_{N}$, where $\mathbf{Z}^{\prime}$ is the combined output, $\mathbf{Z}$ the output due to the signal, and $\mathbf{Z}_{N}$ the output due to the thermal noise from the losses.

If the array is illuminated by a uniform, unpolarized thermal source, and the losses in the array are at the same temperature as the source, $T_{s}$, the travelling waves at the output will be uncorrelated, and appear to originate from a thermal source having temperature $T_{s}$. Under these circumstances, using (16)

$$
\mathbf{Z}^{\prime}=\frac{h \nu}{\exp \left[h \nu / k T_{s}\right]-1} \mathbf{I}_{P}=\frac{h \nu^{3}}{c^{2}} \frac{1}{\exp \left[h \nu / k T_{s}\right]-1} \mathbf{R}+\mathbf{Z}_{N},
$$

where $\mathbf{R}$ has elements

$$
R_{p p^{\prime}}=\int_{\mathcal{A}} \mathbf{t}_{p}^{*}(\hat{\boldsymbol{\Omega}}) \cdot \mathbf{t}_{p^{\prime}}(\hat{\boldsymbol{\Omega}}) d \boldsymbol{\Omega} .
$$

Rearranging,

$$
\mathbf{Z}_{N}=\frac{h \nu}{\exp \left[h \nu / k T_{s}\right]-1}\left[\mathbf{I}_{P}-\frac{1}{\lambda^{2}} \mathbf{R}\right] .
$$

If the synthesised beam patterns are known, and all of the overlap integrals calculated, giving $\mathbf{R}$, the thermal noise and the correlations at the output, $\mathbf{Z}_{N}$, can be found.

Now consider the case where the phased array is not passive, for example when HEMT amplifiers are used, but the noise temperatures of the primary receiving antennas 
are known, and equal. By definition, the noise temperature of a receiver is the temperature that a matched source would need to have in order to generate the same output as a noiseless, but otherwise identical system. Let us call the associated noise correlation matrix at the output $\mathbf{Z}_{N}^{\prime}$, where $\mathbf{Z}^{\prime}=\mathbf{Z}+\mathbf{Z}_{N}^{\prime}$, then to find $\mathbf{Z}_{N}^{\prime}$ we simply need to calculate the coefficients of a uniform sky having the same physical temperature as the noise temperatures of the receivers. Using (14) and (11) we get

$$
\mathbf{Z}_{N}=\frac{h \nu}{\exp \left[h \nu / k T_{N}\right]-1} \frac{1}{\lambda^{2}} \mathbf{R},
$$

where $T_{N}$ is the noise temperature of the individual receivers. In both cases, if the beams are orthogonal, the noise sources are uncorrelated. In the case where the primary receiver temperatures are different, a similar scheme can easily be established.

\section{Fluctuations and Sensitivity}

The last step in the analysis is to determine the fluctuations in power and the correlations between the fluctuations in power at the output ports of a phased array once $\mathbf{Z}$, or $\mathbf{Z}^{\prime}$, is known; after all, it is the fluctuations that ultimately determine the sensitivity of an instrument. Following recent work on multimode detectors [14, 15], it is straightforward to determine the fluctuations in any power-related measurement that is made at the output ports.

If $\mathbf{Z}$ is the coherence matrix of the complex travelling wave amplitudes at the output ports, and $\mathbf{W} \in \mathbb{C}^{P \times P}$ is a matrix that characterises the nature of the measurement being made, the expectation value of the measurement, $\langle P\rangle$, is given by

$$
\bar{P}=\langle P\rangle=\Delta \nu \operatorname{Tr} \mathbf{W Z},
$$

where $\Delta \nu$ is the RF bandwidth. (39) can be appreciated by remembering that the trace of the product of two matrices constitutes an inner product in the abstract vector space of matrices. Thus, (39) describes the way in which the state of coherence of the source projects onto the state of coherence to which the measurement system is sensitive. The structure of (39) has be explored in detail, and leads to the coupled-mode theory of power detection [15, 16, 17].

More significantly, it has also been shown[16] that if two measurements are made, one represented by $\mathbf{W}^{a}$ and one by $\mathbf{W}^{b}$, then the covariance of the measurements, $\operatorname{Cov}\left[P^{a}, P^{b}\right]=\left\langle\left(P^{a}-\bar{P}^{a}\right)\left(P^{b}-\bar{P}^{b}\right)\right\rangle$, is given by

$$
\operatorname{Cov}\left[P^{a}, P^{b}\right]=\frac{\Delta \nu}{\tau}\left[\operatorname{Tr} \mathbf{W}^{a} \mathbf{Z} \mathbf{W}^{b} \mathbf{Z}+\delta_{a b} h \nu_{0} \operatorname{Tr} \mathbf{W}^{a} \mathbf{Z}\right],
$$

where $\nu_{0}$ is the central RF frequency, and $\tau$ the time for which the output is integrated. (40) is valid for thermal sources, and when the integration time is much longer than either the coherence time of the source or the intrinsic response time of the detector. It is valid, therefore, for astronomical phased arrays. The first term in (40) can be identified with the noise associated with classical waves, whereas the second term with the noise associated with photon counting. Thus, (40) contains the transition from fully 
bunched to Poisson statistics as the wavelength of operation moves from the microwave range through into the far infrared. (40) contains a considerable amount of detail, including partition noise, and the randomization of photon arrival times due to losses and imperfect quantum efficiencies.

We can imagine three principle measurements at the output ports. First consider a measurement having the matrix elements

$$
W_{i j}^{a}=\eta^{a} \delta_{i j} \delta_{i a},
$$

where $i, j \in\{1, \cdots, P\}$, which is diagonal with just one non-zero element. Substituting (41) in (39) gives

$$
\begin{aligned}
\left\langle P^{a}\right\rangle & =\Delta \nu \sum_{i j} W_{i j}^{a} Z_{j i} \\
& =\Delta \nu \eta^{a} \sum_{i j} \delta_{i j} \delta_{i a} Z_{j i} \\
& =\Delta \nu \eta^{a} Z_{a a} .
\end{aligned}
$$

(41) therefore corresponds to measuring the expectation value of the power at port $a$, using a detector having quantum efficiency $\eta^{a}$. Likewise, a power measurement at port $b$ is characterised by

$$
W_{i j}^{b}=\eta^{b} \delta_{i j} \delta_{i b}
$$

In the case of a phased array where only powers are measured, which would correspond to an ordinary imaging array with the appropriate synthesised beams, (41) and (43) can be substituted into (40) to give

$$
\operatorname{Cov}\left[P^{a}, P^{b}\right]=\frac{\Delta \nu}{\tau}\left[\eta^{a} \eta^{b}\left|Z_{a b}\right|^{2}+\delta_{a b} h \nu_{0} \eta^{a} Z_{a a}\right]
$$

When we are interested in the true fluctuations in the power at port $a$, as distinct from the fluctuations in the absorbed power, then $a=b$ and $\eta^{a}=\eta^{b}=1$, and the root mean square fluctuation, $\Delta P^{a}$, is given by

$$
\Delta P^{a}=\left\{\operatorname{Cov}\left[P^{a}, P^{a}\right]\right\}^{1 / 2}=\left(\frac{\Delta \nu}{\tau}\right)^{1 / 2}\left[\left|Z_{a a}\right|^{2}+h \nu_{0} Z_{a a}\right]^{1 / 2} .
$$

We can also form the quantity, which is the noise in the measurement normalised by the signal,

$$
\frac{\Delta P^{a}}{\left\langle P^{a}\right\rangle}=\frac{1}{\tau^{1 / 2}}\left[\frac{1}{\Delta \nu}+\frac{h \nu_{0}}{\Delta \nu Z_{a a}}\right]^{1 / 2} .
$$

or

$$
\frac{\Delta P^{a}}{\left\langle P^{a}\right\rangle}=\frac{1}{(\Delta \nu \tau)^{1 / 2}}\left[1+\frac{\Delta \nu}{\bar{n}}\right]^{1 / 2},
$$

where $\bar{n}=\Delta \nu Z_{a a} / h \nu_{0}$ is the average photon rate. In the case where the photon rate is much greater than the bandwidth, or equivalently many photons arrive in the radiation's coherence time, the sensitivity scales according to the radiometer equation; in the case 
where very few photons arrive in the coherence time, the sensitivity scales according to photon counting.

Likewise the root mean square correlation, $\Delta P^{a b}$, between the fluctuations in two different power measurements, $a \neq b$, is given by (44) as

$$
\Delta P^{a b}=\left\{\operatorname{Cov}\left[P^{a}, P^{b}\right]\right\}^{1 / 2}=\left(\frac{\Delta \nu}{\tau}\right)^{1 / 2}\left|Z_{a b}\right|,
$$

and we also have

$$
\frac{\Delta P^{a b}}{\left\langle P^{a}\right\rangle^{1 / 2}\left\langle P^{b}\right\rangle^{1 / 2}}=\frac{1}{(\Delta \nu \tau)^{1 / 2}} \frac{\left|Z_{a b}\right|}{\left(Z_{a a} Z_{b b}\right)^{1 / 2}}=\frac{1}{(\Delta \nu \tau)^{1 / 2}}\left|\Gamma^{a b}\right|,
$$

where $\left|\Gamma^{a b}\right|$ is the correlation coefficient. (49) is the Hanbury Brown-Twiss effect for phased arrays. It does not show Poisson noise because the Poisson term corresponds to photon arrival times that are uncorrelated. In the case where the synthesised beams are orthogonal, and the source uniform, $Z_{a b}=0$, and the fluctuations in the powers measured at different ports are uncorrelated. In the case of non-orthogonal beams, or a non-uniform source on the sky, the fluctuations are correlated, and the correlations must be taken into account when calculating the noise in the reconstructed image.

Now consider the case where we wish to measure the correlations between the travelling waves at the output ports, say $a$ and $b$, for the purpose of recovering an image, as in done in interferometry. In this context, there are two measurement-system matrices of interest:

$$
\begin{aligned}
& R_{i j}^{a b}=\eta^{a b} \frac{1}{2}\left(\delta_{i a} \delta_{j b}+\delta_{i b} \delta_{j a}\right) \\
& I_{i j}^{a b}=\eta^{a b} \frac{i}{2}\left(\delta_{i a} \delta_{j b}-\delta_{i b} \delta_{j a}\right),
\end{aligned}
$$

where $a \neq b$, and the $i$ in the prefactor corresponds to the unit imaginary, rather than the index, with no confusion. The two matrices comprising the elements listed in (50) are Hermitian, and characterise realizable measurements.

Substituting (50) in (39),

$$
\begin{aligned}
& \left\langle R^{a b}\right\rangle=\Delta \nu \eta^{a b} \operatorname{Re} Z_{a b} \\
& \left\langle I^{a b}\right\rangle=\Delta \nu \eta^{a b} \operatorname{Im} Z_{a b},
\end{aligned}
$$

and the two measurements correspond to finding the real and imaginary parts of the correlations between ports $a$ and $b$, which is equivalent to finding the in and out of phase components of the fringe formed when the signals at $a$ and $b$ are combined. More specifically, the matrices correspond to using an analogue correlator to measure the cos and $\sin$ fringes.

We can now substitute (50) into (40) to give

$$
\operatorname{Cov}\left[R^{a b}, R^{a b}\right]=\frac{\Delta \nu}{\tau}\left[\left(\eta^{a b}\right)^{2} \frac{1}{2}\left(Z_{a a} Z_{b b}+\operatorname{Re} Z_{a b}^{2}\right)+h \nu_{0} \eta^{a b} \operatorname{Re} Z_{a b}\right],
$$

giving a noise to signal ratio of

$$
\frac{\Delta R^{a b}}{\left\langle R^{a b}\right\rangle}=\frac{1}{(\Delta \nu \tau)^{1 / 2}} \frac{\left[\frac{1}{2}\left(Z_{a a} Z_{b b}+\operatorname{Re} Z_{a b}^{2}\right)+h \nu_{0} \operatorname{Re} Z_{a b}\right]^{1 / 2}}{\operatorname{Re} Z_{a b}},
$$


for $\eta^{a b}=1$, which corresponds to the actual power. For a completely incoherent source, and orthogonal beams, $Z_{a b}=0$, the noise becomes

$$
\Delta R^{a b}=\frac{1}{(\Delta \nu \tau)^{1 / 2}} \frac{1}{\sqrt{2}}\left(\left\langle P_{a a}\right\rangle\left\langle P_{b b}\right\rangle\right)^{1 / 2}
$$

and the noise is classical, with the fluctuations being proportional to the geometric means of the two power levels, which is a well known feature of noise in interferometers.

In the case where the two signals at $a$ and $b$ are fully coherent, $Z_{a a} Z_{b b}=\left|Z_{a b}\right|^{2}$, we get

$$
\frac{\Delta R^{a b}}{\left\langle R^{a b}\right\rangle}=\frac{1}{\tau^{1 / 2}}\left[\frac{1}{\Delta \omega}+\frac{h \nu_{0}}{\Delta \omega\left|Z_{a b}\right| \cos \theta_{a b}}\right]^{1 / 2},
$$

or

$$
\frac{\Delta R^{a b}}{\left\langle R^{a b}\right\rangle}=\frac{1}{(\Delta \nu \tau)^{1 / 2}}\left[1+\frac{\Delta \nu}{\bar{n}_{c}}\right]^{1 / 2},
$$

where $\bar{n}_{c}$ is the mean number of photons in the in-phase correlated power, and $\theta_{a b}$ is the phase of $Z_{a b}$. Thus, for weak fringes, and even in the nulled region of a strong fringe, classical noise is swapped for Poisson noise. In general, the nature of the noise will change throughout the fringe.

Similarly, we have

$$
\operatorname{Cov}\left[I^{a b}, I^{a b}\right]=\frac{\Delta \nu}{\tau}\left[\left(\eta^{a b}\right)^{2} \frac{1}{2}\left(Z_{a a} Z_{b b}-\operatorname{Re} Z_{a b}^{2}\right)+h \nu_{0} \eta^{a b} \operatorname{Im} Z_{a b}\right],
$$

giving

$$
\frac{\Delta I^{a b}}{\left\langle I^{a b}\right\rangle}=\frac{1}{(\Delta \nu \tau)^{1 / 2}} \frac{\left[\frac{1}{2}\left(Z_{a a} Z_{b b}-\operatorname{Re} Z_{a b}^{2}\right)+h \nu_{0} \operatorname{Im} Z_{a b}\right]^{1 / 2}}{\operatorname{Im} Z_{a b}},
$$

for $\eta^{a b}=1$.

For incoherent travelling waves, the noise is the same as for the in-phase measurement, (54). For fully coherent signals,

or

$$
\frac{\Delta I^{a b}}{\left\langle I^{a b}\right\rangle}=\frac{1}{\tau^{1 / 2}}\left[\frac{1}{\Delta \nu}+\frac{h \nu_{0}}{\Delta \nu\left|Z_{a b}\right| \sin \theta_{a b}}\right]^{1 / 2} .
$$

$$
\frac{\Delta I^{a b}}{\left\langle I^{a b}\right\rangle}=\frac{1}{(\Delta \nu \tau)^{1 / 2}}\left[1+\frac{\Delta \nu}{\bar{n}_{s}}\right]^{1 / 2},
$$

where $\bar{n}_{s}$ is the mean number of photons in the quadrature component of the correlated power. Overall, the same behaviour is seen as for the in-phase component.

Finally, we can correlate the fluctuations in the in-phase and quadrature measurements, giving

$$
\operatorname{Cov}\left[R^{a b}, I^{a b}\right]=\frac{\Delta \nu}{\tau}\left[\left(\eta^{a b}\right)^{2} \frac{1}{2} \operatorname{Im} Z_{a b}^{2}\right]
$$

and

$$
\frac{\Delta R I^{a b}}{\left\langle R^{a b}\right\rangle^{1 / 2}\left\langle I^{a b}\right\rangle^{1 / 2}}=\frac{1}{(\Delta \nu \tau)^{1 / 2}} \frac{1}{\sqrt{2}}\left[\frac{\operatorname{Im} Z_{a b}^{2}}{\operatorname{Re} Z_{a b} \operatorname{Im} Z_{a b}}\right]^{1 / 2},
$$


giving

$$
\frac{\Delta R I^{a b}}{\left\langle R^{a b}\right\rangle^{1 / 2}\left\langle I^{a b}\right\rangle^{1 / 2}}=\frac{1}{(\Delta \nu \tau)^{1 / 2}} .
$$

It is also possible to calculate the correlations between the fluctuations in the real and imaginary parts of fringe measurements on two different pairs of ports.

\section{Conclusion}

We have analyzed the behaviour of phased arrays from a functional perspective, and shown that their operation is intimately related to the mathematical theory of frames. In cases where the beams are non-orthogonal, or even linearly dependent, image reconstruction can be carried out using dual beams. The theory of frames allows one to assess, in a straightforward manner, whether the powers or correlations at the output ports of a phased array contain sufficient information to allow some class of field or intensity distribution to be reconstructed precisely. We have also identified the natural modes of phased arrays, which are important for understanding information throughput and aperture synthesis interferometry.

In order to calculate the behaviour of an imaging phased array it is only necessary to know the synthesised reception patterns, which may be non-orthogonal. It is not necessary to know anything about the internal construction of the array itself. As a consequence, data can be taken from experimental measurements or from electromagnetic simulations. The ability to assess behaviour simply from the synthesised beams separates the process of choosing the best beams for a given application from the process of realizing the beams in practice. It also suggests important techniques for analysing experimental data.

Our model allows the straightforward calculation of quantities such as the correlations in the fluctuations at the output ports of an phased array. Indeed, sources can be constructed through expressions of the kind (15), the correlations at the output ports calculated through (11), or in the modal case (31), system noise can be included through (37) and (38), and the expectation values, fluctuations, and correlations between fluctuations in measurements at the output determined through (39) and (40). The whole procedure only requires simple matrix algebra. The scheme is conceptually and numerically powerful[18, and in an upcoming paper we shall present simulations showing dual beams, natural modes, and image reconstructions.

\section{References}

[1] van Ardenne A, Smolders A and Hampson G 2000 Active adaptive antennas for radio astronomy: Results of the R \& D program towards the square kilometer array Radio Telescopes ed H R Butcher; Proc. SPIE 4015 420-433

[2] Braun R 1997 The concept of the square kilometer array interferometer Proc. High Sensitivity 
Radio Astronomy, ed N Jackson and R J Davies, (Cambridge Univ. Press, Cambridge, UK) 260-268

[3] Ellingson S W 2003 Efficient multibeam synthesis with interference nulling for large arrays IEEE Trans. Antennas Propag. 51 503-511

[4] Ardenne A, Wilkinson P, Patel P, Vaate J 2004 Electronic Multi-beam Radio Astronomy Concept: Embrace a demonstrator for the European SKA program Experimental Astronomy 17 65-77

[5] Padman R Optical fundementals for array feeds 1995 Multi-feed Systems for Radio Telescopes ed DT Emerson and J M Payne (ASP Conf. Series) 75 3-26

[6] Murphy J A and Padman R 1988 Focal plane and aperture plane heterodyne array receivers for millimeter-wave radio astronomy-a comparison Int. J. Infrared Millimeter Waves $\mathbf{9}$ 667-704

[7] Casazza P G The art of frame theory Taiwanese J. Mathematics 4 129-201

[8] Daubechies I 1990 The wavelet transform, time-frequency localization and signal analysis IEEE TRans. Inform. Theory 961-1005

[9] Daubechies I, Grossmann A, and Meyer Y 1986 Painless nonorthogonal expansions J. Math. Phys. 27 1271-1283

[10] Massey R and Refregier A 2005 Polar shapelets Mon. Not. R. Astron. Soc. 363, 197-210

[11] Refregier A 2003 Shapelets:I A method for image analysis Mon. Not. R. Astron. Soc. 338 35-47

[12] Berry R H, Hobson M P and Withington S 2004 Modal decomposition of astronomical images with applications to shapelets Mon. Not. R. Astron. Soc. 354 199-211

[13] Withington S, Hobson M P and Campbell E S 2004 Modal foundations of close-packed optical arrays with particular application to infrared and millimeter-wave astronomical interferometry J. Appl. Phys. 96 1794-1802

[14] Saklatvala G, Hobson M P and Withington S 2006 Simulations of multimode bolometric interferometers Mon. Not. R. Astron. Soc. 367, 1194-1200

[15] Withington S, Campbell E S and Hobson M P 2005 Quantum statistical analysis of multimode far-infrared and submillimetre-wave astronomical interferometers J. Opt. Soc. Am. A 22 19371946

[16] Saklatvala G, Withington S and Hobson M P 2007 A coupled mode theory of far-infrared and submillimetre-wave detectors J. Opt. Soc. Am. A 24 764-775

[17] Withington S and Yassin G 2001 Power coupled between partially coherent vector fields in different states of coherence J. Opt. Soc. Am. A 18 3061-3071

[18] Withington S, Saklatvala G and Hobson M P 2006 Partially coherent analysis of imaging and interferometric phased arrays: noise, correlations, and fluctuations J. Opt. Soc. Am. A 23 13401348 